\title{
The magnetic field of the $\beta$ Lyrae system: Orbital and longer time-scale variability
}

\author{
F. Leone ${ }^{1}$, S. I. Plachinda ${ }^{2}$, G. Umana ${ }^{3}$, C. Trigilio ${ }^{3}$, and M. Skulsky ${ }^{4}$ \\ 1 INAF - Osservatorio Astrofisico di Catania, Città Universitaria, 95125 Catania, Italy \\ 2 Crimean Astrophysical Observatory, Nauchny, Crimea, 334413, Ukraine and Isaac Newton Institute of Chile, \\ Crimean Branch \\ 3 Istituto di Radioastronomia del C.N.R., Stazione VLBI di Noto, C.P. 161 Noto, Italy \\ ${ }^{4}$ Lviv Polytechnic University, 79013 Lviv, Ukraine
}

Received 31 January 2003 / Accepted 25 March 2003

\begin{abstract}
The presence of a magnetic field in $\beta$ Lyrae was firstly suggested by Babcock in 1958 and then confirmed by Skulsky in 1982. This kG-order large-scale organized magnetic field has been neglected in interpreting and modeling the large variety of phenomena presented by $\beta$ Lyrae.

Here, we present circular spectropolarimetry of $\beta$ Lyrae showing that its magnetic field is variable with the orbital period and that it has changed in sign and strength between 1980 and 2000. Unfortunately, there are not enough data to conclude if a longer-time variability is super-imposed on the orbital period variability or if the field changes abruptly.

This magnetic field, to our knowledge, is unique. Since we measured the magnetic field in metal lines of the brightest star of the system, we can conclude that this is the first magnetic B-type giant star. In this case, the magnetic field is significantly different from that of Magnetic Chemically Peculiar stars and the long-time-scale variability suggests the presence of a dynamo. However, we cannot rule out that the magnetic field measured on the brightest star is generated by the accretion disk, or that the magnetic field of the embedded star is so elongated in the orbital plane by the disk that it still has a significant strength even at the companion distance.

In any case, the accretion disk is certainly related to the magnetic field of the $\beta$ Lyrae system. We found that variations of the field in sign and strength corresponded to variations in the disk structure, as it is inferred from photometry and spectroscopy. Also, a magnetized disk explains the observed jet-like outflow from the $\beta$ Lyrae system.
\end{abstract}

Key words. stars: individual: HD 174638 - stars: magnetic fields - stars: binaries: spectroscopic - stars: binaries: eclipsing - stars: chemically peculiar

\section{Introduction}

The spectroscopic and eclipsing binary system $\beta$ Lyrae shows a very complex behavior both from a photometric and a spectroscopic point of view (Sahade 1980; Harmanec et al. 2002). Visible light curves are characterized by a $\sim 1$ mag deep primary minimum and a $\sim 0.4$ mag secondary minimum. Usually this is explained assuming that the star which is in front at the primary minimum is larger and of smaller surface brightness than the star that is behind. In the case of $\beta$ Lyrae system, the cool star would have to be a F5 star, whose characteristic lines are not present in the spectrum and that is not visible in the near infrared. So the photometric behavior of $\beta$ Lyrae was understood only after Huang (1963) suggested that the more massive component is surrounded by a flat and opaque disk.

The brightest component is a B6-8II star (Harmanec 1996) losing mass to a more massive star that Mazzali et al. (1992) classified as an early-B Main-Sequence (MS) star. Starting

Send offprint requests to: F. Leone, e-mail: fleone@ct. astro.it from the orbital solutions by Harmanec \& Scholz (1993), Linnell \& Hubeny (1996) found that the mass of the primary (brightest) and secondary components are 2.9 and $13.1 M_{\odot}$ respectively. Assuming that the secondary star is a MS star with a radius of $6 R_{\odot}$, they modeled the light curves and found that the accretion disk has a radius of $30 R_{\odot}$ and is $6 R_{\odot}$ thick, and that the separation of the two components is $58.35 R_{\odot}$.

The presence of a strong and large-scale organized magnetic field in the $\beta$ Lyrae system has been suggested from the pioneer spectropolarimetric observations by Babcock (1958) and then confirmed by Skulsky (1982).

We found there are several reasons to study the magnetic field of $\beta$ Lyrae. If the magnetic field we observe is really located on the brightest star, it would be the only known B-type giant star with a measurable magnetic field (Borra 1982). Interest in understanding the magnetic field of $\beta$ Lyrae system comes from the high energy phenomena: we know that this system is characterized by X-ray and radio emission (Berghofer \& Schmitt 1994; Umana et al. 2000). Moreover, mass transfer and 
Table 1. Lines and Landé factors used to measure the effective magnetic field of $\beta$ Lyrae at the Crimean Astrophysical Observatory (CAO) and Osservatorio Astrofisico di Catania (OACt).

\begin{tabular}{lr|lr}
\hline \multicolumn{2}{c|}{ CAO } & \multicolumn{2}{|c}{ OACt } \\
Lines & $g_{\text {eff }}$ & Lines & $g_{\text {eff }}$ \\
\hline TiII 4533.969 & 1.100 & SiII 5055.984 & 1.10 \\
FeII 4534.168 & 1.570 & FeII 5260.259 & 1.17 \\
FeII 4541.524 & 0.777 & FeII 5276.002 & 0.91 \\
FeII 4549.474 & 1.030 & FeII 5339.585 & 1.17 \\
SiIII 4552.600 & 1.250 & SII 5432.797 & 1.10 \\
FeII 4555.893 & 1.250 & SII 5453.855 & 1.21 \\
CrII 4558.650 & 1.160 & SII 5606.151 & 1.17 \\
& & SiII 5978.930 & 1,17 \\
& & FeII 6247.557 & 1.03 \\
& & SiII 6371.371 & 1.33 \\
& & FeII 6456.383 & 1.59 \\
\hline
\end{tabular}

accretion disk could be very different than believed up till now, as they have been modeled neglecting the presence of such a strong magnetic field.

\section{Spectropolarimetric observations}

A direct measurement of stellar magnetic fields is based on the polarimetric properties of Zeeman components. Measurements of the circular polarization in spectral lines give the average, over the visible stellar surface, of the longitudinal field, the so called effective magnetic field $\left(B_{\text {eff }}\right)$.

Measurements of $B_{\text {eff }}$ of $\beta$ Lyrae relatively to the 19801988 have been partially published by Skulsky $(1982,1985)$.

In 1993-1995 and 2000, measurements of the longitudinal magnetic field of $\beta$ Lyrae were obtained at the Crimean Astrophysical Observatory with the $2.6 \mathrm{~m}$ Shajn telescope equipped with the achromatic Stokesmeter mounted in front of the entrance slit of the coudé spectrograph (Plachinda \& Tarasova 1999). The spectrograms we used were taken in the spectral region $4530-4565 \AA$, and the resolving power of the spectra was approximately $1.5 \times 10^{4}$. Signal-to-noise ratios varied from 210 to 890 , and the number of individual measurements responsible for the $B_{\text {eff }}$ varied from 4 to 30 depending on the different number of spectral lines used on different nights. The lines used and their Landé factors are listed in Table 1.

Stokes $V$ spectra in the 500-650 nm interval of $\beta$ Lyrae were obtained at the Osservatorio Astrofisico di Catania in 1999. Observations and data reduction procedures are described in Leone et al. (2000). The lines used and their Landé factors are listed in Table 1 . The signal-to-noise ratio was always larger than 200 .

The complete logbook of the observations is given in Table 2, where we report the Julian Data, $B_{\text {eff }}$ (average of values from different metal lines) and its error (standard deviation).
Table 2. Measurements and standard deviations of the effective magnetic field of the $\beta$ Lyrae system are listed according to the year of the observations and observatory: Special Astrophysical Observatory (SAO), Crimean Astrophysical Observatory (CAO) and Osservatorio Astrofisico di Catania (OACt).

\begin{tabular}{|c|c|c|c|c|c|}
\hline \multicolumn{3}{|c|}{ SAO 1980-1988 } & \multicolumn{3}{|c|}{ CAO 1993-1995 + 2000} \\
\hline 44511.200 & -1276 & 186 & 49206.428 & 684 & 146 \\
\hline 44521.250 & -945 & 272 & 49207.304 & -231 & 211 \\
\hline 44829.392 & -953 & 389 & 49208.285 & 862 & 326 \\
\hline 44830.372 & -997 & 149 & 49495.479 & 830 & 565 \\
\hline 44831.332 & -733 & 264 & 49499.483 & 367 & 340 \\
\hline 44832.392 & -1254 & 212 & 49500.380 & -424 & 97 \\
\hline 44834.232 & -1468 & 312 & 49502.421 & 330 & 250 \\
\hline 44835.372 & -2120 & 226 & 49503.414 & -149 & 311 \\
\hline 44836.382 & -1532 & 248 & 49505.416 & -156 & 346 \\
\hline 44837.372 & -1952 & 318 & 49557.354 & 50 & 162 \\
\hline 44838.322 & -1725 & 304 & 49558.360 & 160 & 281 \\
\hline 44839.342 & -1070 & 161 & 49564.371 & 87 & 656 \\
\hline 45181.303 & -1314 & 220 & 49848.495 & 254 & 95 \\
\hline 45183.333 & -1544 & 208 & 49854.509 & 747 & 421 \\
\hline 45184.342 & -1930 & 155 & 49855.494 & 778 & 341 \\
\hline 45185.352 & -1632 & 318 & 49857.488 & 949 & 644 \\
\hline 45190.252 & -916 & 172 & 49955.331 & -172 & 98 \\
\hline 45191.312 & -873 & 135 & 49956.323 & 371 & 114 \\
\hline 45192.252 & -822 & 118 & 49969.283 & -182 & 201 \\
\hline 45193.242 & -976 & 145 & 49970.311 & 550 & 269 \\
\hline 45564.332 & -1221 & 138 & 51764.473 & 224 & 160 \\
\hline 45897.505 & -1739 & 132 & 51765.410 & -242 & 202 \\
\hline 45896.523 & -1775 & 295 & 51766.460 & -437 & 171 \\
\hline 45900.553 & -1082 & 181 & 51767.429 & 317 & 232 \\
\hline 46602.483 & -925 & 109 & 51768.410 & -486 & 422 \\
\hline 46603.463 & -874 & 108 & 51772.433 & 701 & 331 \\
\hline 46604.423 & -1133 & 125 & 51832.285 & -563 & 194 \\
\hline 46605.443 & -1425 & 112 & 51833.287 & -371 & 275 \\
\hline 47346.323 & -1745 & 176 & & & \\
\hline 47347.333 & -1665 & 204 & \multicolumn{3}{|c|}{ OACt 1999} \\
\hline 47348.403 & -1262 & 165 & 51355.556 & 490 & 360 \\
\hline 46605.443 & -1425 & 112 & 51356.523 & 1540 & 490 \\
\hline 47346.323 & -1745 & 176 & 51357.514 & 1400 & 790 \\
\hline 47347.333 & -1665 & 204 & 51358.529 & 1160 & 400 \\
\hline \multirow[t]{9}{*}{47348.403} & -1262 & 165 & 51359.531 & 330 & 870 \\
\hline & & & 51360.545 & 1140 & 400 \\
\hline & & & 51361.537 & 635 & 1010 \\
\hline & & & 51362.517 & 2185 & 860 \\
\hline & & & 51363.514 & 1060 & 810 \\
\hline & & & 51364.522 & 1250 & 660 \\
\hline & & & 51365.573 & 1700 & 475 \\
\hline & & & 51366.482 & 1600 & 790 \\
\hline & & & 51367.490 & 2260 & 730 \\
\hline
\end{tabular}

\section{The observed magnetic field of $\beta$ Lyrae}

Babcock (1958) included $\beta$ Lyrae in the list of stars in which the presence of a magnetic field is probable but not firmly established. 
From Skulsky (1985), it is known that the effective magnetic field of $\beta$ Lyrae system, as measured in 1980-1988, was sinusoidally variable with the orbital period. It presented a $-1.2 \mathrm{kG}$ average value and $0.47 \mathrm{kG}$ amplitude. Assuming that this field belongs to the primary component and from analogy with Magnetic Chemically Peculiar (MCP) stars where the field is frozen on the stellar surface, Skulsky (1985) interpreted the periodic variability as due to a de-centered dipole $(a=0.08)$ whose axis is tilted with respect to the rotational axis by $28^{\circ}$.

To phase our measurements of the effective field, we have adopted the orbital ephemeris determined by Harmanec \& Scholz (1993):

$$
\begin{aligned}
\mathrm{JD}(\text { Primary eclipse })= & 2408247.966+12.91378 \times E \\
& +3.87196 \times 10^{-6} \times E^{2} .
\end{aligned}
$$

We measured a $B_{\text {eff }}$ changing significantly:

- In 1993-1995, the field was mainly positive (Fig. 1). The average value and standard deviation of our measurements are 0.40 and $0.44 \mathrm{kG}$ respectively.

- In 1999, the field was always positive with an average value equal to 1.3 and standard deviation of $0.65 \mathrm{kG}$.

- Even though the orbital phase coverage was incomplete in 2000 , we observed again negative values.

The variability of the magnetic field with the orbital period, observed in the 1980 's, is not so clearly present in the later data. Based on Stellingerwerf's (1978) method as it is coded in the IRAF package, a period analysis does not support the orbital period at all. Formally the best period is 1.73 days for the 1993-1995 data and 2.49 days for the 1999 measurements. Both values are different from the 4.73 day period found by Harmanec et al. (1996) from the $\mathrm{H} \alpha$ and $\mathrm{HI} 6678 \AA$ radial velocities. Unfortunately, we do not have enough data to conclude if a longer time periodic variability is super-imposed on the orbital period variability or if the field abruptly changes.

The properties of the $\beta$ Lyrae magnetic field are very different from those observed in MCP stars. In this class of stars the magnetic field is observed to be stable on time scales of several decades. Consider the first star observed to be a magnetic star, 78 Vir in 1947 by Babcock (1947): its magnetic field appears unchanged up to 1999 (Leone \& Catanzaro 2001). The same is true for all MCP stars discovered by Babcock in the 1950's. Moreover, for MCP stars the magnetic field variation is commonly interpreted as due to a mainly dipolar configuration. In the case of the $\beta$ Lyrae system, it is much more difficult to recover the magnetic field geometry. The brightest component is filling its Roche-lobe and is not spherical.

\section{Location and origin of the magnetic field}

We have observed the effects of a magnetic field on the spectral lines of the brightest component of the $\beta$ Lyrae system, i.e. the B8II star. We could conclude that this is the first B-type giant whose magnetic field has been measured with certainty. The variability with orbital period implies a synchronization between the orbital and rotational periods. Moreover the magnetic field variation in intensity and sign on a longer time scale could indicate a dynamo origin of the field.

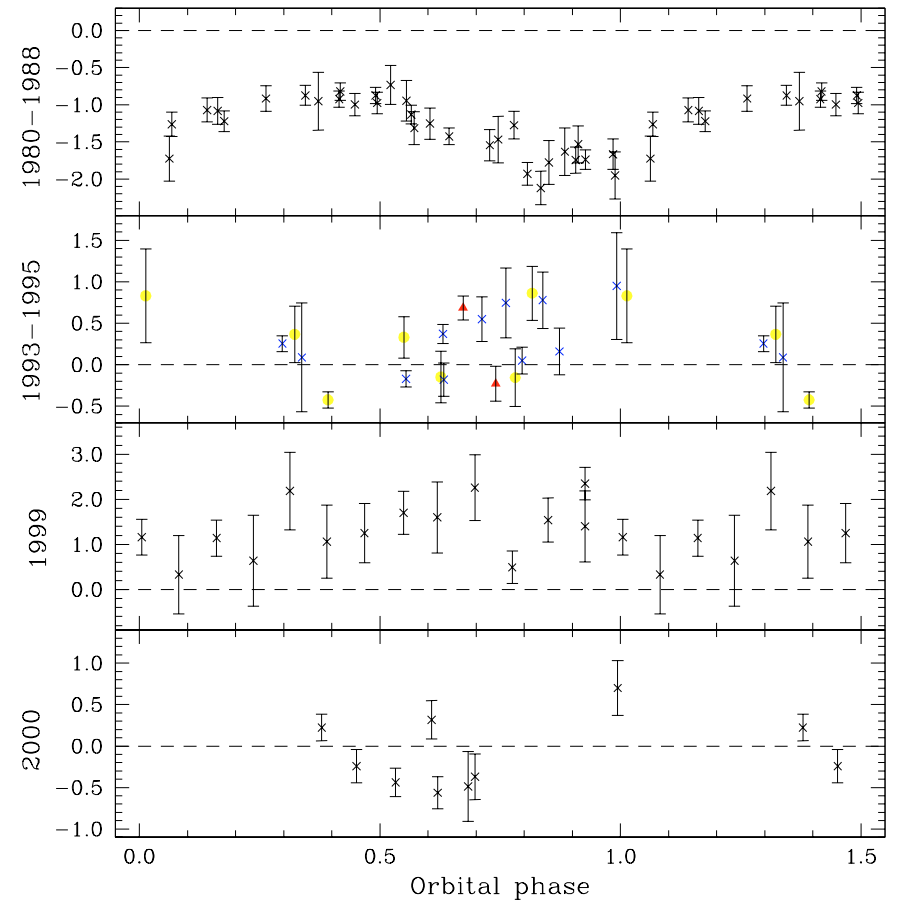

Fig. 1. Effective magnetic field in $\mathrm{kG}$ of $\beta$ Lyrae measured in 19801988, 1993-1995, 1999 and 2000. Data are folded with the orbital period reported by Harmanec \& Scholz (1993). Dashed lines represent the null value. Among the 1993-1995 data, triangles represents 1993, circles the 1994 and crosses the 1995 observations respectively. Error bars are equal to two times the standard deviation, see text.

However, the importance of the accretion disk in generating and modifying external magnetic fields cannot be neglected. We find that dynamo-active accretion disks can be at the origin of some phenomena presented by the $\beta$ Lyrae system. First of all there is the jet-like outflow discovered by Harmanec et al. (1996) with optical interferometry and confirmed by Hoffman et al. (1998) with linear spectropolarimetry. This outflow is necessary to explain the radio-nebulae spatially resolved by Umana et al. (2000). According to numerical simulations, a magnetized disk may drive a magnetic wind, providing a good explanation for the origin of the collimated structures (Casse \& Keppens 2002).

Stone \& Norman (1994) have shown that the magnetic field of an accretion disk is intrinsically unsteady. As to the $\beta$ Lyrae system, it is known that the accretion disk is not stationary: a) The light curves are not stable, they vary in shape and amplitude (Guinan 1989), especially during the minima (Wilson 1974). The primary minimum of the visible light-curve, which occurs when the disk is in front of the primary star, changes in deepness and shape from epoch to epoch and even from one cycle to the next (Sahade 1980). Van Hamme et al. (1995) concluded that the long-term variations of the light curve have a 283.4-day period. b) According to Batten \& Sahade (1973), the $\mathrm{H}_{\alpha}$ emission can be considered as the superposition of a narrow component presenting small or no Radial Velocity (RV) changes and a broad component that moves out of phase with respect to the RV of the brightest star. These authors also found 
secular changes of the emission strength. $c$ ) Some strongly (200-300 km) shifted lines (satellite lines) are observed only during the primary minimum and are attributed to the disk rotating around the massive star (Sahade et al. 1980). We find that the long-time-scale variability of the magnetic field is related to the variability of the disk structure. Particularly important for us is Skulsky's (1993) finding that the behavior of the SiII 6347 and 6371 A satellite lines changed abruptly from 1991 to 1992. It appears that the satellite lines changed just after the 19801988 campaign, when we observed an always negative effective magnetic field, and before the 1993-1995 when the field appeared mainly positive. Moreover, Struve (1958) found that during the primary minimum the SiII lines of the primary star can show very different radial velocities even in two successive cycles. These velocity changes are explained in terms of different amounts of electron scattering due to a variable density of the disk (Sahade et al. 1980). Moreover, Skulsky (1992) measured the Doppler shift of characteristic features in the $\mathrm{H}_{\alpha}$ and HeI $6678,7065 \AA$ line profiles continuously from 1985 to 1991, and concluded that clear differences appeared from 1990 in the radial velocity of the short-wavelength peak, also noticeable for the center emission.

If we measure on the brightest star of the $\beta$ Lyrae system the magnetic field generated by the accretion disk, the variability with orbital period is not a surprise. It appears that the average of the magnetic field over the visible surface of the brightest star changes with the orbital motion because of the field gradient. On the hemisphere of the brightest star facing the disk, the magnetic field is stronger than in the opposite hemisphere. Moreover, the brightest star, transferring matter onto the companion, could not be spherical. Thus the visible photosphere changes in shape and area with the orbital phase and along with it the effective magnetic field, average over the visible surface of longitudinal components.

\section{Conclusions}

We have performed spectropolarimetric measurements of the effective magnetic field of the $\beta$ Lyrae system between 1993 and 2000. We found that the magnetic field as measured from metal lines of the brightest component is of $\mathrm{kG}$ order, and it changes with the orbital period, and on a longer time scale. Our data are not sufficient to conclude if this field changes periodically or abruptly. We therefore encourage any observational campaign able to cover at least the long time variability observed for the light curves ( $\sim 300$ days) with the aim to understand if the magnetic field also presents long time scale periodic variability or if the variations are abrupt.

Assuming that the magnetic field belongs to the brightest star, the straightforward conclusion is that this is the first B-type giant whose magnetic field has been measured with certainty. In this case, the magnetic field variability with the orbital period is indicative of rotation-orbit synchronism and the longtime variability could be related to the dynamo period.
However, we cannot rule out that we measure the field generated in the accretion disk on the brightest component. The most recent numerical simulations by von Rekowski et al. (2003) shown that a dynamo-active accretion disk can drive an outflow even without any external magnetic field. We therefore do not need to conclude that at least one of the components of $\beta$ Lyrae is a magnetic star. Moreover, solutions of the dynamo-active accretion disks foresee a significant magnetic field in the orbital plane which in the case of $\beta$ Lyrae could be the one measured on the brightest component. The presence of a magnetized disk easily explains the jet-like outflow presented by $\beta$ Lyrae and the fact that the disk is non-stationary.

$\beta$ Lyrae is listed in the General catalogue of $A p$ and Am stars by Renson et al. (1991). Because of the long time variability superimposed on the orbital period, we concluded that it is not a main-sequence magnetic chemically peculiar star.

We also conclude that further modeling of $\beta$ Lyrae massloss or disk should include the presence of the observed magnetic field.

\section{References}

Babcock, H. W. 1947, ApJ, 105, 105

Babcock, H. W. 1958, ApJS, 3, 141

Batten, A. H., \& Sahade, J. 1973, PASP, 85, 599

Berghofer, T. W., \& Schmitt, J. H. M. M. 1994, A\&A, 292, L5

Borra, E. F. 1982, ARA\&A, 20, 191

Casse, F., \& Keppens, R. 2002, ApJ, 581, 988

Guinan, E. F. 1989, Space Sci. Rev., 50, 35

Harmanec, P., \& Scholz, G. 1993, A\&A, 279, 131

Harmanec, P., Morand, F., Bonneau, D., et al. 1996, A\&A, 312, 879

Harmanec, P. 2002, Astron. Nachr, 323, 87

Hoffman, J. L., Nordsieck, K. H., \& Fox, G. K. 1998, AJ, 115, 1576

Huang, S. 1963, ApJ, 138, 342

Leone, F., Catanzaro, G., \& Catalano, S. 2000, A\&A, 355, 315

Leone, F., \& Catanzaro, G. 2001, A\&A, 365, 118

Linnell, A. P., \& Hubeny, I. 1996, ApJ, 471, 958

Mazzali, P. A., Pauldrach, A. W. A., Puls, J., \& Plavec, M. J. 1992, A\&A, 254, 241

Plachinda, \& Tarasova, T. N. 1999, ApJ, 514, 402

Renson, P., Gerbaldi, M., \& Catalano, F. A. 1991, A\&AS, 89, 429

Sahade, J. 1980, Space Sci. Rev., 26, 349

Skulsky, M. Yu. 1982, Pis'ma Astron. Zh., 8, 238

Skulsky, M. Yu. 1985, Pis'ma Astron. Zh., 11, 51

Skulsky, M. Yu. 1992, Pis'ma Astron. Zh., 19, 114

Skulsky, M. Yu. 1993, Pis'ma Astron. Zh., 19, 417

Stellingwerf, R. F. 1978, ApJ, 224, 953

Stone, J. M., \& Norman, M. L. 1994, ApJ, 433, 746

Struve, O. 1958, PASP, 70, 585

Umana, G., Maxted, P. F. L., Trigilio, C., et al. 2000, A\&A, 358, 229

Van Hamme, W., Wilson, R. E., \& Guinan, E. F. 1995, AJ, 110, 1350

von Rekowski, B., Brandeburg, A., Dobler, W., \& Shukurov, A. 2003, A\&A, 398, 825

Wilson, R. E. 1974, ApJ, 189, 319 